\title{
Estimating Fetal Gestational Age Using Cardiac Valve Intervals
}

\author{
Faezeh Marzbanrad $^{1,2}$, Ahsan H Khandoker ${ }^{2,3}$, Yoshitaka Kimura ${ }^{4}$, Marimuthu Palaniswami ${ }^{2}$, Gari D \\ Clifford ${ }^{5,6}$ \\ ${ }^{1}$ Monash University, Clayton, VIC, Australia \\ 2 The University of Melbourne, Parkville, VIC, Australia \\ ${ }^{3}$ Khalifa University of Science, Technology and Research, Abu Dhabi, UAE \\ 4 Tohoku University, Sendai, Japan \\ ${ }^{5}$ Department of Biomedical Informatics, Emory University, Atlanta, GA USA \\ ${ }^{6}$ Department of Biomedical Engineering, Georgia Institute of Technology, Atlanta, GA, USA
}

\begin{abstract}
A new automated method for estimation of the gestational age is presented in this paper based on the intervals between fetal cardiac valve timings and the $Q$-wave of fetal electrocardiogram (fECG). The intervals were estimated automatically from one-dimensional Doppler Ultrasound and noninvasive fECG. Among the intervals, Isovolumic Contraction Time, Electromechanical Delay Time, Ventricular Filling Time and their interactions were selected in a stepwise regression process. Compared with Crown-Rump Length as gold standard, a mean absolute error of 3.8 weeks was obtained using leave-one-out cross validation. This method also outperformed a fetal heart rate based approach. Since valve intervals reflect the autonomic function, this method provides a novel measure of the development of fetal autonomic nervous system that may be growth curve independent.
\end{abstract}

\section{Introduction}

Estimation of the gestational age (GA) is crucial for antenatal diagnosis, monitoring fetal growth, predicting the delivery date and management of pre- and post-term pregnancies. The established gold standards are obstetric ultrasound measures, which provide accurate GA estimation but are affected by genetic variations, inherent variability of growth, unsuitable positioning of the fetus and operator error $[1,2]$. For example, Crown-Rump Length (CRL) which is one of the most precise ultrasound measures, has 95\% prediction interval of around 10 days [3]. High equipment cost, lack of skilled sonographers or physicians also limit the use of ultrasound in low income countries. Therefore alternative techniques are needed as an adjunct or substitute where ultrasound methods are unavailable or inadequate due to pathologies, unsuitable positioning or techni- cal issues.

one promising alternative GA estimator is Fetal Heart Rate (FHR), which is affordable and applicable without special skills, hence feasible for low income countries [4-6]. Various FHR variability (FHRV) parameters were found closely related to the fetal maturation $[5,7]$. A more critical indication for estimating GA is assessing the development of the fetal brain which may affect the entire life. Functional Fetal Autonomic Brain Age Score (fABAS) has been recently introduced which uses FHR patterns for multivariate analyses of universal developmental indices [5]. However, FHR is influenced by arrhythmias, fetal behavioral states, heart rate patterns and maternal conditions $[8,9]$.

Fetal cardiac valve intervals are other measures which could be obtained from non-invasive, low cost and easyto-operate devices, and provide reliable markers for fetal development and wellbeing $[10,11]$. These intervals are based on opening and closing timings of the fetal cardiac valves and can be automatically estimated as proposed in our previous papers $[11,12]$. The valve intervals can be used to assess the Autonomic Nervous System (ANS) function, as an alternative to FHRV, since the cardiac mechanics are known to reflect the autonomic control $[13,14]$. Significant changes in the valve intervals with advancing GA were also reported in our previous studies [11]. The aim of the current paper is to use these intervals as a novel GA estimator which reflects the development of fetal ANS.

\section{Methods}

\subsection{Data acquisition and fetal ECG extrac- tion}

One-dimensional Doppler Ultrasound (1-D DUS) data were obtained using ultrasonic transducer 5700 (Coromet- 


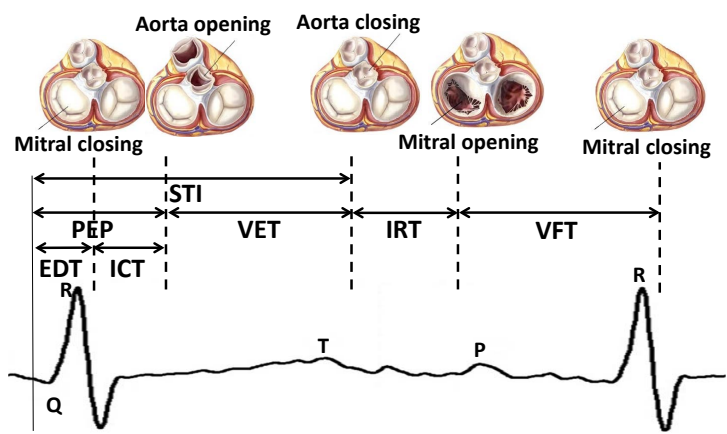

Figure 1. An illustrative example of fetal cardiac intervals: Systolic Time Interval (STI), Electromechanical Delay Time (EDT), Isovolumic Contraction Time(ICT), PreEjection Period (PEP), Ventricular Ejection Time (VET), Isovolumic Relaxation Time (IRT), Ventricular Filling Time (VFT).

rics, model: 116) with $1.15 \mathrm{MHz}$ signals. Data were collected from 57 pregnant women at the GA of 16 to 41 $(33 \pm 6)$ weeks with healthy single pregnancies at Tohoku University Hospital in Japan. The GA was estimated by measuring CRL at about 10 weeks using ultrasound imaging by a trained sonographer. Abdominal ECG signals were recorded simultaneous with DUS using 12 electrodes. All DUS and ECG recordings were one minute in length, sampled at $1 \mathrm{kHz}$ with 16-bit resolution. To extract fetal Electrocardiogram (fECG) from the composite abdominal signal, a combination of maternal ECG cancellation and blind source separation with the reference signal (BSSR) was used [15]. Fetal QRS locations were detected by the modified Pan and Tompkins peak detection described in [16].The study protocol was approved by Tohoku University Institutional Review Board and written informed consent was obtained from all participants. More details can be found in our previous study [15].

\subsection{Estimation of cardiac valve intervals}

Cardiac valve intervals illustrated in figure 1, were obtained based on the fECG Q-wave and the opening and closing of the valves detected from the high frequency component of the DUS signal. The valve motion events were detected using a model-based method which was proposed in our previous paper and summarized as follows [12]. The envelope of the signal decomposed by wavelet transform at scale 2, was segmented into cardiac cycles (R-R intervals), normalized and clustered into 6 patterns using K-means. The aim was to find the following events and attribute to the peaks of the components: Aortic valve opening (Ao), Aortic valve closing (Ac), Mitral opening (Mo), Miral closing (Mc) and four transitional event between them (T1 to T4). A hybrid Support Vector Machine-
Hidden Markov Model (SVM-HMM) was trained for each cluster separately, using time and amplitude of the peaks of the signal as features. The training and validation were based on simultaneous fetal echo-cardiography images and expert annotations. Each segment of the normalized envelope of the detail signal was attributed to one of the clusters which gave the minimum Euclidean distance to the centroid of the cluster. Then the sequence of events attributed to the peaks of the signal were identified by the Viterbi algorithm, using the trained SVM-HMM specific to the corresponding cluster. More details about this method can be found in our previous paper [12].

\subsection{Estimation of the gestational age}

Three sets of parameters were used to estimate the GA: - Valve-timing parameters: From the parameters shown in figure 1, EDT, ICT, VET, IRT and VET were selected. Only PEP and STI were excluded as they were linearly related to other intervals.

- FHR-related parameters: Time and frequency domain FHRV parameters were used including: Mean and standard deviation of RR intervals (mRR and SDRR), Root Mean Square of the Successive Differences (RMSSD) between adjacent RR intervals, low frequency (LF: 0.03-0.15 $\mathrm{Hz}$ ) which is related to the neural sympathetic activity, medium frequency (MF: 0.15-0.5 Hz) corresponding to the fetal movements and maternal breathing, high frequency (HF: 0.5-1 Hz) which marks the presence of fetal breathing, the ratio $\mathrm{LF} /(\mathrm{MF}+\mathrm{HF})$ and total power (TP). More details can be found in [17].

- Combined model: Combined valve timing and FHRrelated parameters.

In order to predict the GA from these parameters a stepwise regression analysis was employed based on individual and all combinations of parameters and the models including an intercept, linear, squared terms and cross-products. Stepwise regression automatically adds to or removes from the model in a forward and backward process to determine a final model, using an F-test applied to the sum of the squared error before and after adding a parameter $(p<0.05)$ as the criterion for including a parameter. Root Mean Squared Error, R-squared, adjusted R-squared and the F-test statistic results versus constant model were calculated for the regression of each set of parameters. An average leave one out cross validation error in GA estimation was calculated. The difference between the CRL-based and regression-based GA estimate was made at every stage to provide an estimate of out of sample performance.

\section{Results}

Using fetal heart valve timings as parameters, stepwise regression resulted in the following model: 
Table 1. Results of Stepwise regression using valve intervals, including estimated coefficients and their Standard Error (SE), t-statistic and p-value for the F-statistic.

\begin{tabular}{lllll}
\hline \hline & Estimate & SE & t-test & p-value \\
\hline Intercept & -276.810 & 61.772 & -4.481 & $4.218 * 10^{-5}$ \\
EDT & 5.496 & 1.215 & 4.525 & $3.641 * 10^{-5}$ \\
ICT & 7.897 & 1.743 & 4.530 & $3.574 * 10^{-5}$ \\
VFT & 0.682 & 0.267 & 2.551 & 0.014 \\
EDT*ICT & -0.140 & 0.034 & -4.142 & $1.295 * 10^{-4}$ \\
ICT*VFT & -0.017 & 0.007 & -2.273 & 0.027 \\
\hline \hline
\end{tabular}

Estimated GA $=a_{0}+a_{1} E D T+a_{2} I C T+a_{3} V F T+$ $a_{4} E D T * I C T+a_{5} I C T * V F T$

where $a_{0}, a_{1}, \ldots, a_{5}$ are the coefficients, GA is in weeks and the intervals are in milliseconds. Table 1 shows the estimate and Standard Error (SE) of coefficients, t-statistic to test the null hypothesis that each coefficient is zero given the other predictors in the model and F-statistic (p-value) for the null hypothesis.

An F-test on the regression model versus constant model showed significance of the model (F-statistics 15.1, pvalue $\left.=4.36 * 10^{-9}\right)$. Standard deviation of the error distribution was 4.01 (weeks) and R-squared and adjusted RSquared were $60 \%$ and $56 \%$, respectively.

The following regression model using FHRV parameters were obtained using stepwise regression:

Estimated GA $=b_{0}+b_{1} m R R+b_{2} S D R R$.

where $b_{0}, b_{1}$ and $b_{2}$ are the coefficients, GA is in weeks and the parameters are in milliseconds. Only mean and standard deviation of fetal RR-intervals significantly contributed to the model. Table 2 shows the estimate and Standard Error (SE), t-statistic and F-statistic p-value for the coefficients, similar to table 1.

The statistics for the F-test on the regression model versus constant model was significant (F-statistics 6.08, pvalue $=0.004)$. However the SD of the error distribution was 5.55 (weeks) which was larger than the SD for the model with valve intervals, and R-squared and adjusted Rsquared were only $18 \%$ and $15 \%$, respectively, which were smaller than those of the model with valve intervals.

Using leave one out cross validation, the mean absolute difference between the CRL-estimated age and the GA estimated from the proposed model was found to be 5.1 weeks using FHRV parameters and 3.8 weeks using valve timing intervals. When attempting to select a combined model, none of the FHR parameters were selected and therefore did not provide any additional value or increase the GA estimation accuracy.

\section{Discussion and Conclusion}

In this paper a new GA estimator using fetal cardiac valve intervals is proposed. These intervals were estimated automatically from the raw recordings, hence less affected
Table 2. Results of Stepwise regression using FHRV parameters, including estimated coefficients and their Standard Error (SE), t-statistic and p-value for the F-statistic.

\begin{tabular}{lllll}
\hline \hline & Estimate & SE & t-test & p-value \\
\hline Intercept & 4.788 & 10.866 & 0.441 & 0.661 \\
mRR & 0.064 & 0.026 & 2.432 & 0.018 \\
SDRR & 0.120 & 0.058 & 2.044 & 0.046 \\
\hline \hline
\end{tabular}

by human errors compared to sonography. Valve intervals were obtained using easy to handle devices and with less required skill to operate compared to sonography. It is also possible to use only 1-D DUS to obtain ICT, VET, IRT and VFT as described in our previous paper [18]. Since 1-D DUS device can cost as little as $\$ 17$ and can be performed by nonexperts with limited training, it can be used to estimate the GA in resource limited settings [6].

The complex interplay between autonomic control of the heart and cardiac mechanics characterized by the valve intervals, has been previously reported in literature and is consistent with the results of this study $[13,14]$. According to the literature, PEP is attributed to the sympathetic influences on the heart [13]. As shown in figure 1, PEP (Qwave to aorta opening) is comprised of two intervals: EDT (Q-wave to mitral closing) and ICT (mitral closing to aorta opening). The results show that not only ICT and EDT, but also their interaction significantly contributes to the GA estimation. As shown in in table 1, VFT was also selected as a contributing term. Although less emphasis has been placed on VFT than other intervals in the literature and clinical practice, studies on adults found that VFT is controlled by autonomic tone, which is the balance between sympathetic and parasympathetic activity [19]. Therefore fetal development can be assessed by these intervals which evolve concomitantly with the changes in sympathetic and parasympathetic activities during fetal maturation.

Fetal autonomic brain age can be assessed using FHRV parameters [5,7]. Results of this paper showed that the new method based on valve intervals outperforms the FHRbased method in estimating the GA although only time and frequency domain parameters and non identical populations were used. FHR is influenced by other factors such as behavioral states of the fetus and maternal physiological and psychological conditions $[8,9]$. However, the recordings used in this study are short and may not thoroughly represent the FHRV patterns which are used to evaluate the brain development [5]. Longer recordings will enable a better comparison with FHRV patterns to assess the development of autonomic control and evaluation of the influence of behavioral states. The proposed techniques also need to be tested on pathological fetal conditions such as Intrauterine Growth Restriction (IUGR) in future studies. Results of this study showed that using valve intervals, the GA can be estimated with comparable accuracy to the ex- 
isting methods. This proposed method could be improved by assessing the quality of Doppler and fECG signals. It should be also noted that the error was obtained by comparing against the estimated GA using CRL as gold standard, while CRL itself is subject to error (95\% prediction interval is around 10 days)[3], particularly in case of unsuitable positioning .

In conclusion, the proposed GA estimation method resulted in 3.8 weeks error compared to CRL age and outperformed the GA estimation by FHRV parameters. It can be performed using easy to operate devices and requires less skills compared to sonography methods. Considering that the valve intervals reflect the autonomic control of the fetal heart, the new method provides assessment of the fetal ANS development, which could be growth curve independent (since it reflects neural development, not physical size). As a result the method might provide indications of IUGR early in pregnancy and potentially lead to early interventions.

\section{References}

[1] Campbell S, Warsof SL, Little D, Cooper DJ. Routine ultrasound screening for the prediction of gestational age. Obstetrics Gynecology 1985;65(5):613-620.

[2] Hadlock F, Deter R, Harrist R, Park S. Fetal abdominal circumference as a predictor of menstrual age. American Journal of Roentgenology 1982;139(2):367-370.

[3] Grange G, Pannier E, Goffinet F, Cabrol D, Zorn JR. Dating biometry during the first trimester: accuracy of an everyday practice. European Journal of Obstetrics Gynecology and Reproductive Biology 2000;88(1):61-64.

[4] Tezuka N, Saito H, Hiroi M. Comparison of the accuracy of gestational age estimation from fetal heart rate and crownrump length. Primary Care Update for ObGyns 1998; 5(4):193.

[5] Hoyer D, Tetschke F, Jaekel S, Nowack S, Witte OW, Schleußner E, Schneider U. Fetal functional brain age assessed from universal developmental indices obtained from neuro-vegetative activity patterns. PloS One 2013; 8(9):e74431.

[6] Stroux L, King N, Fathima S, Hall-Clifford R, Rohloff P, Clifford GD. A low-cost perinatal monitoring system for use in rural Guatemala. In Appropriate Healthcare Technologies for Low Resource Settings (AHT 2014). IET, $2014 ; 1-4$.

[7] Van Leeuwen P, Geue D, Lange S, Hatzmann W, Grönemeyer D. Changes in the frequency power spectrum of fetal heart rate in the course of pregnancy. Prenatal Diagnosis 2003;23(11):909-916.

[8] Mantel R, van Geijn HP, Ververs IA, Copray FJ. Automated analysis of near-term antepartum fetal heart rate in relation to fetal behavioral states: The Sonicaid System 5000. American Journal of Obstetrics and Gynecology 1991;165(1):57-65.

[9] Monk C, Fifer WP, Myers MM, Sloan RP, Trien L, Hurtado
A. Maternal stress responses and anxiety during pregnancy: effects on fetal heart rate. Developmental Psychobiology 2000;36(1):67-77.

[10] Shakespeare S, Crowe J, Hayes-Gill B, Bhogal K, James D. The information content of Doppler ultrasound signals from the fetal heart. Medical and Biological Engineering and Computing 2001;39(6):619-626.

[11] Marzbanrad F, Kimura Y, Funamoto K, Sugibayashi R, Endo M, Ito T, Palaniswami M, Khandoker AH. Automated estimation of fetal cardiac timing events from Doppler ultrasound signal using hybrid models. Biomedical and Health Informatics IEEE Journal of 2013;18(4):1169-1177.

[12] Marzbanrad F, Kimura Y, Funamoto K, Oshio S, Endo M, Sato N, Palaniswami M, Khandoker A. Model-based estimation of aortic and mitral valves opening and closing timings in developing human fetuses. Biomedical and Health Informatics IEEE Journal of 2014;20(1):240-248.

[13] Cacioppo JT, Berntson GG, Binkley PF, Quigley KS, Uchino BN, Fieldstone A. Autonomic cardiac control. II. noninvasive indices and basal response as revealed by autonomic blockades. Psychophysiology 1994;31(6):586-598.

[14] Di Rienzo M, Vaini E, Castiglioni P, Merati G, Meriggi P, Parati G, Faini A, Rizzo F. Wearable seismocardiography: Towards a beat-by-beat assessment of cardiac mechanics in ambulant subjects. Autonomic Neuroscience 2013; 178(1):50-59.

[15] Sato M, Kimura Y, Chida S, Ito T, Katayama N, Okamura K, Nakao M. A novel extraction method of fetal electrocardiogram from the composite abdominal signal. Biomedical Engineering IEEE Transactions on 2007;54(1):49-58.

[16] Behar J, Oster J, Clifford GD. Non-invasive FECG extraction from a set of abdominal sensors. In Computing in Cardiology Conference (CinC), 2013. IEEE, 2013; 297-300.

[17] Signorini MG, Magenes G, Cerutti S, Arduini D. Linear and nonlinear parameters for the analysisof fetal heart rate signal from cardiotocographic recordings. Biomedical Engineering IEEE Transactions on 2003;50(3):365-374.

[18] Marzbanrad F, Kimura Y, Endo M, Palaniswami M, Khandoker AH. Automated measurement of fetal isovolumic contraction time from Doppler ultrasound signals without using fetal electrocardiography. In Computing in Cardiology Conference (CinC), 2014. IEEE, 2014; 485-488.

[19] Frazier SK, Moser DK, Schlanger R, Widener J, Pender L, Stone KS. Autonomic tone in medical intensive care patients receiving mechanical ventilation and during a cpap weaning trial. Biological Research for Nursing 2008; 9(4):301-310.

Address for correspondence:

Faezeh Marzbanrad

Department of Electrical and Computer Systems Engineering, Monash University, Clayton VIC 3168 Australia

faezeh.marzbanrad@monash.edu 